\title{
Characterization of valuable compounds from winter melon (Benincasa hispida (Thunb.) Cogn.) seeds using supercritical carbon dioxide extraction combined with pressure swing technique
}

\begin{abstract}
In this study, we describe the extraction of different valuable compounds from winter melon seeds using supercritical carbon dioxide extraction combined with pressure swing technique (SCE-PST). The effects of the extraction variables, namely pressure, holding time (HT), and continuous extraction time (CT), were optimized by response surface methodology (RSM) to maximize the crude extraction yield (CEY). The optimal conditions were at pressure of $181.35 \mathrm{bar}$, HT of $9.93 \mathrm{~min}$, and CT of $50.14 \mathrm{~min}$. Under these conditions, the experimental CEY was $235.70 \pm 0.11 \mathrm{mg} \mathrm{g}-1$ with a relatively strong antioxidant activity $(64.42 \pm 0.21 \%$ inhibition of DPPH radicals, $67.36 \pm 0.34 \%$ inhibition of ABTS + radicals) and considerable amount of phenolic compounds $(42.77 \pm 0.40 \mathrm{mg}$ gallic acid equivalent/g extract). The high-performance liquid chromatography (HPLC) analysis revealed that the bioactive phenolic compounds increased significantly using PST $(\mathrm{p}<0.05)$, where gallic acid had the highest concentration $(0.688 \pm 0.34 \mathrm{mg} \mathrm{g}-1)$. The extract obtained using optimal SCE-PST conditions contained more than $83.65 \%$ total unsaturated fatty acids (UFAs) and linoleic acid accounted for $67.33 \pm 0.22 \%$ in the total extract. From the results, the SCE efficiency in terms of extract quantity and quality has been enhanced significantly applying PST. Finally, the results were compared with previous published findings using supercritical carbon dioxide, ultrasound-assisted, and Soxhlet extraction. It was found that higher CEY could be achieved using Soxhlet extraction even through the quality of SCE-PST extracts in terms of antioxidant activity and phenolic compounds was better.
\end{abstract}

Keyword: Antioxidant activity; HPLC; Phenolic compounds; SCE-PST; UFA; Winter melon 